\title{
What the Fetal Face can tell Us: A Discussion of the Evidence, Implications and Potential for Further Research
}

Nadja Reissland

\begin{abstract}
This paper reviews findings in fetal development research using two-dimensional and four-dimensional ultrasound imaging and how these techniques have been applied to increase understanding of the fetus. The limitations of differences in language and methods used to code and score images between research groups will also be explored, reaching the conclusion that a reliable coding scheme for fetal facial movements is essential. Furthermore, applications of the new technology studies of bonding between parent and fetus, cross-cultural research on fetal facial development and medical applications are discussed.
\end{abstract}

Keywords: Fetal facial movements, Ultrasound visualization, 2D/4D scanning.

How to cite this article: Reissland N. What the Fetal Face can tell Us: A Discussion of the Evidence, Implications and Potential for Further Research. Donald School J Ultrasound Obstet Gynecol 2014;8(4):336-343.

Source of support: Nil

Conflict of interest: None

\section{INTRODUCTION}

Developmental changes in patterns of movement of the human fetus and specifically fetal facial movements are still not fully described. ${ }^{1}$ However, with the advent of in utero imaging, particularly four-dimensional (4D) imaging (4D ultrasonography), there is now a means for capturing the repertoire of fetal facial movements and how the display of these movements evolves from the first to third trimester of pregnancy. The age when facial expressions begin to emerge and which of these facial expressions predominate in fetal life are questions that remain unanswered so far. ${ }^{2}$ Whilst a great deal of empirical weight has been given to the idea that developmental plasticity occurs largely in utero $^{3}$, and fetal facial movements have been viewed as a window into the developing nervous system, ${ }^{4}$ the question still remains as to whether or not these fetal

Senior Lecturer

Department of Psychology, Durham University, Science Site Durham, United Kingdom

Corresponding Author: Nadja Reissland, Senior Lecturer Department of Psychology, Durham University, Science Site South Road, Durham DH1 3LE, United Kingdom, e-mail: n.n.reissland@durham.ac.uk facial expressions vary depending on maternal mental and physical health and might reflect the health of the developing fetus. Ultrasonography could help to answer questions including whether fetal imaging can have further use in informing us about the existence of cultural differences in fetal development and whether it may prove helpful in promoting maternal-fetal attachment. In this paper, imaging techniques used in order to chart the development of fetal facial movements are discussed. It is argued that such techniques might have the potential for deriving diagnostic tools aimed at the early detection of developmental dysfunction. ${ }^{5-7}$ If these outcomes are to be met, there needs to be careful consideration of how fetal behaviors and specifically fetal facial expressions are described and analyzed. Both the image quality and the systems used to specify fetal facial movements affect the data derived from in utero imaging and thus the interpretation of the data gained.

Ultrasonography used to assess fetal well-being is now able to produce three-dimensional (3D)/4D images through which fine-grained fetal facial movements can be assessed, in addition to two-dimensional (2D) images that continue to be useful in demonstrating general body movements. The quality of $2 \mathrm{D}$ and 3D/4D images of diagnostic ultrasound examinations differs as they are based on two different modes of acquisition. ${ }^{8}$ 2D processing algorithms are based on pixels within a single image plane. In contrast 3D/4D imaging is based on voxels using information from a number of planes. ${ }^{8}$ Both methods of imaging have been useful in research, but the information gained from them and subsequent interpretations differs.

Results from ultrasound imaging research need to be communicated between different researchers, languages and laboratories. There is currently no common language to describe fetal facial movement, with the result that differences between findings are difficult to resolve or explain. As an example, emotionally charged terminology has been used to label individual muscle movements, leaving coding open to influence by individuals' interpretations of emotional facial signals. A mouth movement could be labeled as 'smile' by one lab but a 'grimace' by another. To identify a face as 'smile' or 'grimace' is a value judgment implying an emotion rather than an objective description of the muscles 
used to make that movement. Recognition of this issue is not new. Ekman and Friesen ${ }^{9}$ argued in agreement with researchers before them that studies of adult facial movements need to reliably identify facial expressions and should not be based on the interpretation of facial expressions in terms of emotional labels. Observers need to reliably label identifiable movements of the facial muscles that form these expressions.

Similarly, discrepancies in relation to fetal activity trajectories over the three trimesters of pregnancy have been attributed to a lack of consistency in approaches to data coding. ${ }^{10}$ Some have reported that fetuses are less active as birth approaches ${ }^{11}$ whereas others find the contrary. ${ }^{12}$ These discrepancies might have to do with the definition of movement themselves or the way these movements are counted, either as frequency of movements or duration of these movements in relation to the time observed. Research also needs to account for factors that may affect fetal behavior, such as the timing of observations as some fetuses are reported to show more activity during the day and others are more active during the night. More needs to be done to understand the impact of such factors.

This review of human fetal facial movements will explore differences between $2 \mathrm{D}$ and $3 \mathrm{D} / 4 \mathrm{D}$ ultrasound scanning techniques in order to resolve variations in findings based on image quality as well as the language and methods used to code and score images. It will also explore the potential impact that fetal imaging research can have if these issues were resolved.

\section{D vs 3D/4D ULTRASOUND IMAGING}

\section{Use of 2D Imaging}

Sonographic examinations over the last 30 years have been conducted in $2 \mathrm{D}$ displays ${ }^{13}$ providing useful information for both medical and research purposes. For example, Little et $\mathrm{al}^{14}$ observed spontaneous and elicited startle behavior in 2D images of fetuses scanned with conventional 2D ultrasound machine with a $3.5 \mathrm{MHz}$ curvilinear scan head. They found no difference between fetal startle behaviors of mothers who consumed alcohol and those who did not. In another paper by the same lab, Hepper et $\mathrm{al}^{15}$ using 2D scanning techniques report that maternal alcohol consumption delays the emergence of the fetal startle responses only for a defined period after which the differences in fetuses of mothers who consume alcohol and those who do not disappear. Together, these papers have offered a useful insight into the effect of alcohol on fetal development. However, only whole body movements rather than specific facial expressions were reported. Regarding fetal facial movements, eye blink and mouth opening have been recorded from data gained from 2D imaging. However, 2D scans cannot be used to test fine grained movements, such as different types of mouth opening as have been distinguished using 4D scans. ${ }^{16}$

De Vries and Fong ${ }^{17}$ used 2D scans to investigate general (nonfacial) fetal movements in addition to examining jaw opening, yawning and eye blink. They argued that their own studies carried out with 2D scans are more useful than $4 \mathrm{D}$ imaging because $4 \mathrm{D}$ imaging can only report a selected area of interest since the whole fetus cannot be visualized at the later gestations. However, these criticisms can also be leveled against 2D scans since in later gestations neither 2D nor 3D/4D scan can visualize the entire fetus.

Unlike in 4D scans, soft tissue is poorly imaged in 2D scans. Rotten and Levaillant ${ }^{18}$ demonstrated the drawback of this in their review of 2D and 4D imaging use in prenatal cleft lip detection. Although both scan types were useful in the detection of cleft palate, cleft lip was more difficult to discern in 2D scans, with low detection rates of $21 \%$ and $30 \%$ compared to an $88 \%$ detection rate in studies using all three planes of the face. This demonstrates that, although 2D scans have their place in detecting some anatomical features, others are underreported.

\section{Use of 3D/4D Imaging}

Investigating fine-grained facial movements enables further insight into fetal development. For this reason, the importance of more advanced 3D/4D scanning technology is paramount. Although 2D scanning has been able to look at some specific facial movements, far more can be identified in 4D images. For example, Reissland et $\mathrm{al}^{19}$ have identified 19 movements in $4 \mathrm{D}$ scans. A number of studies ${ }^{20,21}$ have examined the development of various facial movements using 4D scanning, usually during the late second to third trimester of pregnancy, and have been able to identify specific movements. Kurjak et $\mathrm{al}^{21}$ found that 'smiling' and 'scowling' can easily be distinguished in fetuses as young as 13 weeks up to 33 weeks gestational age, and Hata et $\mathrm{al}^{22}$ demonstrated that, at 20 to 38 weeks of gestation, different types of mouth movement, including 'yawning', 'a little opening', 'chewing' and 'subtle lip movement' were distinguishable from one another.

3D/4D images have advantages besides enabling the detection of fine grained movements. A number of studies have shown that 3D ultrasound might be faster and more accurate compared with 2D sonographic diagnosis. ${ }^{23}$ Benacerraf ${ }^{24}$ argued that the advantage of $3 \mathrm{D}$ imaging is the fact that the acquired volume can 
be displayed in an infinite number of 2D scan planes including planes in which direct image acquisition is not possible. The three orthogonal planes acquired are stored and can be subsequently manipulated so that a number of views are displayed allowing an evaluation of the region of interest. Especially, the c-plane or orthogonal plane in the reconstructed image helps, e.g. in terms of measuring the nuchal translucency, especially in fetuses who in the normal 2D view would lie in a position not allowing such measurements. Additionally, examining the anatomy of the fetus, such as the fetal ear, is helped by 3D surface rendering of the fetal image. Benacerra $\mathrm{f}^{24}$ suggested that $3 \mathrm{D}$ volume scanning opened up new avenues for measuring fetuses, including volume measurements of organs.

Not only 3D imaging facilitates a view of the fetal anatomy but also real-time 3D ultrasound imaging with a temporal component (i.e. 4D ultrasound) is most useful in showing fetal movements in general and fetal facial movements in particular allowing inferences on neuronal functioning. For example, 4D images have been used to identify a number of movements, including yawning compared with mouth opening. These types of movements can be used to infer neural functioning. Reissland et $\mathrm{al}^{16}$ suggest that although the relationship between the neural network of mouth, tongue and respiratory movements is currently unknown in humans, based on research in rats, the function of yawning in fetuses might lie in activity-dependent brain maturation. ${ }^{25}$

Recently, attempts have been made to improve the imaging quality produced through $3 \mathrm{D} / 4 \mathrm{D}$ scanning. ${ }^{8} \mathrm{~A}$ comparison of obstetric image quality on a three-point visual analog scale from one worst to three best images found that the unprocessed image was considered worse compared to a processed 2D image, and a processed 3D image showed the best quality with better depiction of edges and reducing speckle. ${ }^{8}$ Hence, Forsberg et $\mathrm{al}^{8}$ concluded that perception of image quality is significantly enhanced with new image processing methods. These methods will improve as the techniques for image enhancement develop. Currently, HDlive is considered to be the highest standard of image rendering. Rendering images can create a more smooth appearance and possibly make them more attractive to parents. As a research tool, however, the rendering smoothes out aspects of the image necessary for the coding of fetal facial movements and hence is not ideal for coding fine grained fetal facial movements. An illustrative example (Figs 1A and B) demonstrates the comparison of the same fetal face once in HDlive and the next frame in $4 \mathrm{D}$ scanning shows that, in HDlive, the forehead of the fetus is rendered and appears smooth in contrast $4 \mathrm{D}$ scanning visualizes fetal eyebrow movement.

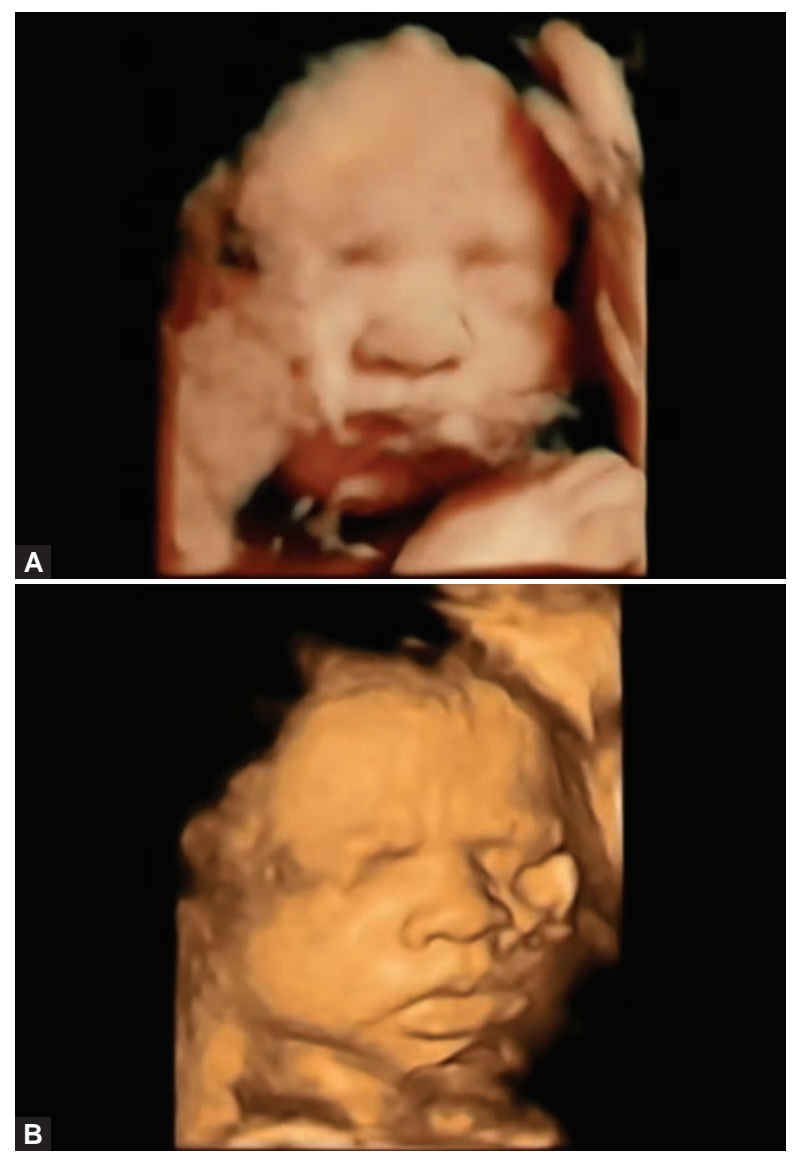

Figs 1A and B: HDlive (A) and 4D ultrasound (B) images illustrating that $4 \mathrm{D}$ ultrasound image show more detail of fetal facial muscle movements

\section{THE NECESSITY OF A FINE-GRAINED CODING SYSTEM}

Despite a relatively large body of research investigating fetal development through ultrasound imaging, there is little standardization in movement definitions for the purpose of coding. As an example, Sato et $\mathrm{al}^{26}$ report $4 \mathrm{D}$ ultrasound studies of fetal facial expressions by fetuses aged between 20 and 24 weeks. They observed the occurrence of isolated movements: mouth movements, yawning, smiling, tongue expulsion, scowling, sucking and blinking. The definitions of these movements are supported by illustrative pictures, but very little information on definitions of these movements is given. This is just one example of how lack of information regarding coding prevents real comparisons between data.

A seminal report of 12 fetal movements by De Vries et al, ${ }^{27,28}$ including jaw opening, startle, breathing movement, head rotation, general movements, etc. was based on 12 fetuses scanned for 60 minutes. They observed scans of fetuses from 7 to 15 weeks gestation and coded these real-time observations. Given the relative large number of movements observed, coding them in real time, rather than off-line, meant that it is likely that some of the movements were missed. 
De Vries et $\mathrm{al}^{27,28}$ proceeded to record the fetal scans at 17 and 19 weeks on videotape and to code fetal motility during playback of the video. Fetal facial movements, specifically jaw openings were found to increase over time. Fetal body stretches and yawns were recorded under one code which means that the results cannot be compared to other studies in which yawns were analyzed separately from fetal trunk and arm and head movements. Furthermore, De Vries and Fong ${ }^{17}$ using 2D imaging reported that jaw openings could be observed from 10 weeks and yawning could be observed from 11 weeks gestation. They did not define yawning as opposed to jaw opening and as a result their data cannot be compared across studies. In a review of publications on fetal yawning, Reissland et $\mathrm{a}^{16}$ observed that groups of researchers came to different conclusions. Some claimed that yawning can be observed prenatally, ${ }^{29}$ whereas others ${ }^{30}$ believed that yawning reported in the literature could be a mouth opening or even a set of repeated mouth openings rather than yawns. One group of investigators vaguely defined yawning, e.g. simply referring to mouth opening and the subjective impression given of a yawn; others were more precise and gave details of the ratio of mouth opening to closing. ${ }^{31}$ Therefore, in order to allow comparisons between results, it is necessary not only to distinguish precisely between different types of mouth movement but also the dynamics of these movements. ${ }^{16}$

Although this research usefully establishes that the fetus does show face, lip and eye movements, comparisons across studies are impossible. Some ${ }^{32}$ have provided very specific definitions of movements by basing the definition of a 'smile' on an anatomically based descriptions [namely Action Unit 12 as defined in $\left.\left(\mathrm{FACS}^{9}\right)\right]$ and in so doing noted that most smiles were observed in fetuses ranging between 160 and 180 days conceptual age. However, the majority of the literature has failed to be so objective in its descriptions. Kawakami and Yanaihara ${ }^{32}$ also defined the condition that the length of observation of this movement was more than one second and could not be attributed to a known external cause in order to further promote objectivity.

\section{USE OF ULTRASOUND IMAGES}

\section{Can We use Fetal Facial Movements for Medical Diagnostics?}

2D imaging has been used for several decades to investigate fetal well-being. Some researchers ${ }^{33}$ suggest that $3 \mathrm{D} / 4 \mathrm{D}$ ultrasound of the fetal face does not aid diagnosis; however, others ${ }^{18,34}$ have concluded that during routine screening when using 2D imaging techniques, the examiner needs to image several planes and hence making the examination longer than with $3 \mathrm{D} / 4 \mathrm{D}$ scanning. Specifically, if using 2D imaging techniques to establish the presence of facial abnormalities of lip and nose morphology (facial dysmorphology) both the midsagittal plane and the anterior 'nose-mouth' coronal plane need to be visualized. The examination of the fetal face for potential anomalies needs to be based on the visualization of three planes which is what $3 \mathrm{D} / 4 \mathrm{D}$ sonography is able to achieve. Specifically, 3D/4D ultrasound imaging allows rapid screening and more precise evaluation of different facial features. ${ }^{35}$ Benacerraf et $\mathrm{al}^{35}$ found that mean time to perform a complete anatomical ultrasound examination of fetuses between 17 and 21 weeks in 2D scanning lasted 19.6 minutes, whereas performing the same task in 3D ultrasound examinations lasted only a mean of 1.8 minutes. Reduction of exposure time is desirable and has been recommended by the British Medical Ultrasound Society ${ }^{36}$ (e.g. BMUS recommends the ALARA (as low as reasonably achievable or minimum necessary exposure) principle.

Kurjak et $\mathrm{al}^{37,38}$ have developed a coding system using 4 D scanning, which is known as KANET. They were able to correlate findings of the KANET with the ATNAT ${ }^{39}$ developed by Amiel-Tison. ${ }^{40,41}$ The ATNAT and KANET examine mostly general body movements. Here, I only focus on aspects of the fetal face which are of interest to this review. Regarding facial movements, the following movements are coded in the KANET: isolated eye blink, grimace, tongue expulsion, mouth opening, yawning (coded under the summary term 'facial alterations'). The ATNAT codes in relation to mouth movements, nonnutritive sucking and ocular signs as present or absent or facial paralysis; fasciculation of the tongue. Given these relatively few facial movements with variations in definitions of the exact movements, it seems unlikely that the positive correlations found in the ATNAT and $\mathrm{KANET}^{39}$ are based on similarities in fetal facial movements. Therefore, if one wants to use fetal facial movements in order to chart fetal well-being, defining more specific facial movements is imperative. Such a well-defined system might be of potential benefit in order to assess fetal and neonatal neurological development.

Campbell ${ }^{42}$ wrote in an editorial that the development of $3 \mathrm{D}$ ultrasound imaging has resulted in a number of publications reaching the conclusion that $3 \mathrm{D} / 4 \mathrm{D}$ produces better images than 2D imaging. Yet, Campbell ${ }^{42}$ also argued that the research on the benefits is still ongoing and not yet conclusive. He recognized that fetal anomalies can be detected by high resolution 2D ultrasound and suggests that the reason additional 3D scanning is not advocated could be related to the increased time in training, including training the sonographer in $3 \mathrm{D} / 4 \mathrm{D}$ scanning modalities. Indeed, with appropriate training, 3D ultrasound has been shown to be 
superior in demonstrating superficial fetal defects, such as facial clefts and studies from several groups have shown that the technique has a high sensitivity for diagnosing defects of the secondary palate which are rarely detected by $2 \mathrm{D}$ ultrasound. ${ }^{43}$

Recently, specific developmental abnormalities have been found to be detectable through $4 \mathrm{D}$ imaging. For example, facial paralysis, a condition involving severe or complete loss of the motor function of facial muscles has been observed prenatally.

Fetal micrognathia is an abnormal development of the mandible. Luedders et $\mathrm{al}^{44}$ suggested that normal development of the mandible depends on mandibular movement during intrauterine development. According to Terzis and Anesti, ${ }^{45}$ this condition has been observed in newborns with reports ranging from 1.4 up to 64 per 1000 birth. Given the importance of this condition, Luedders ${ }^{44}$ argued for 3D ultrasound visualization, which facilitates the identification of facial anomalies in planes that cannot be obtained by conventional 2D imaging. This multiplanar navigation, which is only possible with 3D technology, provides valuable information by enabling the visualization of fetal facial structures from multiple perspectives. ${ }^{2,46}$ Borenstein et $\mathrm{al}^{47}$ reported that using 3D volumes of the fetal head in order to define the relative position of the maxilla and mandible in fetuses with trisomy 18 compared to chromosomally normal individuals improves the detection rate to $92 \%$. Although fetal alcohol syndrome refers to facial dysmorphisms, 48 research has not reported variations in fetal facial movements. Given the changes in the facial anatomy, there is the potential to establish not only the dysmorphism by analyzing the fetal facial anatomy but also variations in movements. This is strong support for a role of $4 \mathrm{D}$ scanning in prenatal diagnosis of anatomical defects.

Furthermore, there is evidence that other indicators of fetal well-being may be detectable through 4D scanning. Fetal stress, allied with maternal stress, is associated with negative perinatal outcomes ${ }^{49,50}$ and may have long-term implications. ${ }^{51,52}$ Significant levels of specific behaviors have been found in fetuses where the mother reports high levels of stress at the time of scanning. For example, the fetuses of mothers reporting stress have been found to show more left handed touches. ${ }^{53}$ This adds further weight to the claim that ultrasound images of the fetus can give an insight into development in utero. ${ }^{54}$

\section{Use of Ultrasound Images to promote Maternal Bonding with the Fetus}

Campbell ${ }^{42}$ suggested that $4 \mathrm{D}$ ultrasound images increase the perception of the 'humanity' of the fetus and thereby might encourage maternal-fetal bonding. A number of publications claim that maternal attachment is increased by $4 \mathrm{D}$ visualization of the fetal face. Notably, a recent review by De Jong-Pleij ${ }^{55}$ suggests that although most studies found an increase in maternal bonding after 4D ultrasound examinations with good visibility, there was no significant difference in terms of degree of maternal bonding after either 3D/4D or 2D scans. Hence, the better visibility of the fetal face in 4D scanning seemed not to add to the boding experience. However, none of these studies specify what aspects of the face the examiner pointed out to the mothers. Did they point to anatomical similarities between mother, father, siblings, grandparents or aunts and uncles? Characteristic shapes of mouth, nose or eyebrows are often well recognized in scans or perhaps a father's smile might be reflected in the mouth movements of the fetus. The importance of $2 \mathrm{D} / 4 \mathrm{D}$ images in relation to attachment and bonding need to be tested with a defined protocol explaining the scan image to the parent.

Other studies examining bonding in relation to fetal scans have analyzed the quality of the image. However, the quality is assessed by the examiner rather than the pregnant woman. ${ }^{56}$ Given that even high quality scans can be difficult to interpret by a naïve observer, quality assessments by the examiner tell us little about what the mother saw in the scan. We suggest it is, therefore, essential to include in the protocol that the examiner points out head, eyes, nose, mouth, tongue and ears of the fetus in order to help the observer to recognize the fetus and to improve the consistency of the interpretation of the scan by the mother.

Given present positive indicators of 4D imaging promoting bonding, further research is needed to explore the significance of this effect. Protocol factors that could increase this effect particularly need to be investigated before conclusion can be reached on the practical implications of 2D and 4D scans for promoting bonding.

\section{Use of Ultrasound Images to provide Information on Cross-cultural Fetal Development}

Research acknowledges the influence of both nature and nurture on development. ${ }^{57}$ It is unclear, however, whether there are differences in fetal development, specifically facial movement development, between cultures given the vague definitions of what a movement represents. Nicoletto and Rinaldi ${ }^{58}$ argue that the uterine environment affects the fetus with both physical factors, such as maternal nutrition and maternal emotional factors, such as maternal stress believed to have a negative impact. Given the apparent sensitivity of the fetus to the environment, we propose that there is potential for fetal 
development to be affected by cross-cultural differences. Differences in developmental patterns of behavior have been found between cultures. For example, although Myowa-Yamakoshi and Takeshita ${ }^{59}$ did not find developmental patterns in fetal self-touch between 24 and 31 weeks gestation in a sample of Japanese fetuses. Reissland et $\mathrm{al}^{53}$ observed fetuses born in the United Kingdom and found that as fetuses matured from 24 to 36 weeks of gestation, they increasingly touched the mouth region and lower part of their face.

Research on cross-cultural differences in fetal behavior is, to our knowledge, restricted largely to early infancy and does not include fetal facial behaviors. In that regard, one study by Di Pietro et $\mathrm{al}^{60}$ is of interest. Although not analyzing fetal facial movements, they examined fetuses in two very different cultures, namely Baltimore (Maryland, USA) and Lima, (Peru). They measured fetal heart rate and fetal movements. In both measures, one being very well defined and the other being less clear, they found differences. Baltimore fetuses exhibited a greater number of movements, but Lima fetuses spent more of the observation period moving. However, Di Pietro et $\mathrm{al}^{60}$ point out that, even though pregnant women were matched on social class, education, body mass index and all had healthy pregnancies delivering healthy babies, 'disparities in motor development are dependent on the manner in which motor activity is defined'. They speculate that differences detected between different cultures are dependent on processes shaping fetal development that are mediated through biological and physiological aspects of the pregnancy in these two cultures. However, without precise measures, it remains speculation which needs to be tested.

\section{SUMMARY}

This review argues that $4 \mathrm{D}$-scanning techniques may be more suited to investigating fetal facial movements then either 2D imaging or HDlive techniques. Irrespective of the way, the fetal face has been visualized, research advances have been hampered to some extent by a missing common language in coding facial expressions. Such a coding system allowing comparison between studies is essential in order to further the scientific study of fetal facial development as an index of fetal healthy development. Such a common language would also facilitate novel fields of research including the study of cross-cultural similarities and differences in fetal development. Only one study ${ }^{60}$ examined differences in general movements based on an actograph between two different cultures, namely Peru (Lima) and the US (Baltimore) and found significant differences.
There is no published cross- cultural research on fetal facial movements which might indicate differences in development and hence this needs a collaborative effort between various cultural setting using comparable scanning techniques as well as the same definitions of fetal facial movements. Here, we highlight the necessity of the development of a fine grained and reliable coding system to enable effective comparison of data regarding fetal facial movements between laboratories. If we are to extend this understanding to a cross-cultural understanding of fetal development, such a coding system is of even greater importance in order to enable effective cross-cultural collaboration.

Notwithstanding these problems in comparative data, 3D/4D ultrasound as it develops will facilitate the collection of important medical as well as social information which ultimately might lead to better health behaviors mediated by attachment to the fetus not only by mothers but also the whole family which can follow the progress of fetal development and learning about the personality of the fetus before birth.

\section{ACKNOWLEDGMENTS}

I thank Louisa and Toto for their comments on a previous draft.

\section{REFERENCES}

1. De Vries JI, Hopkins B. Fetal movements and postures: what do they mean for postnatal development? In Prenatal Development of Postnatal Functions. In: Hopkins B, Johnson SP, editors. Praeger Publishers: Westport; 2005. p. 177-219.

2. Kurjak A, Miskovic B, Andonotopo W, Stanojevic M, Azumendi G, Vrcic H. How useful is 3D and 4D ultrasound in perinatal medicine? J Perinat Med 2007;35(1):10-27.

3. Barker DJP. The origins of the developmental origins theory. J Intern Med 2007;261:412-417.

4. Hopkins B, Reissland N. Epilogue. Inf Child Dev 2010;19(1): 125-126.

5. Kurjak A, Carrera JM, Stanojevic M, Andonotopo W, Azumendi G, Scazzocchio E, Medic M, Salihagic-Kadic A. The role of $4 \mathrm{D}$ sonography in the neurological assessment of early human development. Ultrasound Rev Obstet Gynecol 2004;4(3):148-159.

6. Di Pietro JA. Neurobehavioral assessment before birth. Mental Retar Develop Disabil Res Rev 2005;11(1):4-13.

7. Hata T, Yanagihara T, Matsumoto M, Hanaoka U, Ueta M, Tanaka Y, Kanenishi K, Kuno A. Yamashiro C, Tanaka $\mathrm{H}$, et al. Three-dimensional sonographic features of fetal central nervous system anomaly. Acta Obstet Gynecol Scand 2000;79:635-639.

8. Forsberg F, Berghella V, Merton DA, Rychlak K, Meiers J, Goldberg BB. Comparing image processing techniques for improved 3-dimensional ultrasound imaging. J Ultrasound Med 2010;29(4):615-619.

9. Ekman P, Friesen W. Facial Action Coding System: a technique for the measurement of facial movement. Consulting Psychologists Press, Palo Alto; 1978. 
10. Ten Hof J, Nijhuis IJM, Mulder EJH, Nijhuis JG, Narayan H, Taylor DJ, Visser GH, Mulder EJ. Quantitative analysis of fetal generalized movements: methodological considerations. Early Hum Dev 1999;56(1):57-73.

11. Ten Hof J, Nijhuis IJM, Mulder EJH, Nijhuis JG, Narayan H, Taylor DJ, Westers P, Visser GH. Longitudinal study of fetal body movements: nomograms, intrafetal consistency and relationship with episodes of heart rate patterns A and B. Pediatr Res 2002;52(2):568-575.

12. Patrick J, Campbell K, Carmichael L, Probert C. Influence of maternal heart rate and gross fetal body movements on the daily pattern of fetal heart rate near term. Am J Obstet Gynecol 1982;144(5):533-538.

13. Benacerraf BR, Shipp TD, Bromley B. How sonographic tomography will change the face of obstetric sonography. J Ultrasound Med 2005;24(3):371-378.

14. Little JF, Hepper PG, Dornan JC. Maternal alcohol consumption during pregnancy and fetal startle behaviour. Physiol Behav 2002;76:691-694.

15. Hepper PG, Dornan JC, Lynch C, Maguire JF. Alcohol delays the emergence of the fetal elicited startle response, but only transiently. Ultrasound Obstet Gynecol 2004;23(3):224-231.

16. Reissland N, Francis B, Mason J. Development of fetal yawn compared with non-yawn mouth openings from 24-36 weeks gestation. PLoS ONE 2012;7(11):e50569, doi: 10.1371/journal. pone.0050569.

17. De Vries JIP, Fong BF. Normal fetal motility: an overview. Ultrasound Obstet Gynecol 2006;27(6):701-711.

18. Rotten D, Levaillant JM. Two- and three-dimensional sonographic assessment of the fetal face: a systematic analysis of the normal face. Ultrasound Obstet Gynecol 2004;23(3): 224-231.

19. Reissland N, Francis B, Mason J, Lincoln K. Do facial expressions develop before birth? PLoS ONE 2011;6(8):e24081, doi: 101371/journal.pone.0024081.

20. Yan F, Dai SY, Akther N, Kuno A, Yanagihara T, Hata T. Fourdimensional sonographic assessment of fetal facial expression early in the third trimester. Int J Gynecol Obstet 2006;94(2): 108-113.

21. Kurjak A, Azumendi G, Vecek N, Kupesic S, Solak M, Varga D, Chervenak F. Fetal hand movements and facial expression in normal pregnancy studied by four-dimensional sonography. J Perinat Med 2003;31(6):496-508.

22. Hata T, Kanenishi K, Akiyama M, Kimura K. Real-time 3D sonographic observation of fetal facial expression. J Obstet Gynaecol Res 2005;31(4):337-340.

23. Bega G, Lev-Toaff A, Kuhlman K, Kurtz A, Goldberg B. Wapner R. Three-dimensional ultrasonographic imaging in obstetrics: present and future. J Ultrasound Med 2001;20(4):391-408.

24. Benacerraf BR. Three-dimensional fetal sonography: use and misuse. J Ultrasound Med 2002;21(10):1063-1067.

25. Khazipov R, Sirota A, Leinekugel X, Holmes GL, Ben-Ari Y, Buzsaki G. Early motor activity drives spindle bursts in the developing somatosensory cortex. Nature 2004 Dec 9;432: 758-761.

26. Sato M, Kanenishi K, Hanaoka U, Noguchi J, Marumo G, Hata T. 4D ultrasound study of fetal facial expressions at 20-24 weeks of gestation. Int J Gynecol Obstet 2014;126(3):275-279.

27. De Vries JIP, Visser GHA, Prechtl HFR. The emergence of fetal behaviour. II: quantitative aspects. Early Hum Dev 1985;12(2): 99-120.
28. De Vries JIP, Visser GHA, Prechtl HFR. The emergence of fetal behaviour. I: qualitative aspects. Early Hum Dev 1982;7(4): 301-322.

29. Sherer DM, Smith SA, Abramowicz JS. Fetal yawning in utero at 20 weeks' gestation. J Ultrasound Med 1991;10(2):68.

30. McMagnus B, Devine P, Brandsetter RD. A fetal yawn? New Engl J Med 1997 May;336:1329.

31. Petrikovsky B, Kaplan G, Holsten N. Fetal yawning activity in normal and high-risk fetuses: a preliminary observation. Ultrasound Obstet Gynecol 1999;13(2):127-130.

32. Kawakami F, Yanaihara T. Smiles in the fetal period. Infant Behav Develop 2012;35:466-471.

33. Newnham JP, Evans SF, Michael CA, Stanley FJ, Landau LI. Alcohol delays the emergence of the fetal elicited startle response, but only transiently. Lancet 1993;342:887-891.

34. Tan S, Ipek A. Detailed ultrasound screening in the second trimester: pictorial essay of normal fetal anatomy. J Clin Ultrasound 2012;40:280-300.

35. Benacerraf BR, Shipp TD, Bromley B. Three-dimensional US of the fetus: volume imaging. Radiology 2006;238(3):988-996.

36. British Medical Ultrasound Society (BMUS http:// fetalanomaly.screening.nhs.uk/fetalanomalyresource/ images / stories / Downloads / 2.5.1_safe_environment / bmus-safety-guidelines-2009-revision-final-nov-2009.pdf downloaded 30 August 2014).

37. Kurjak A, Miskovic B, Stanojevic M, Amiel-Tison C, Ahmed B, Azumendi G, Vasilj O, Andonotopo W, Turudic T, Salihagic-Kadic A. New scoring system for fetal neurobehavior assessed by three- and fourdimensional sonography. J Perinat Med 2008;36(1): 73-81.

38. Kurjak A, Abo-Yaqoub A, Stanojevic M, Yigiter BA, Vasilj O, Lebit D, Shaddad AN, Ahmed B, Kavak ZN, Miskovic B, et al. The potential of $4 \mathrm{D}$ sonography in the assessment of fetal neurobehavior: multicentric study in high-risk pregnancies. J Perinat Med 2010;38(1):77-82.

39. Miskovic B, Vasilj O, Stanojevic M, Ivanković DD, Kerner M, Tikvica A. The comparison of fetal behavior in high risk and normal pregnancies assessed by four dimensional ultrasound. J Matern Fetal Neonat Med 2010;23(12):1461-1467.

40. Amiel-Tison C. Clinical assessment of the infant nervous system. In: Levene ML, Chervenak FA, Whittle M, editors. Fetal and Neonatal Neurology and Neurosurgery. 3rd ed. London: Churchill Livingstone; 2001. p. 99-120.

41. Amiel-Tison C. Update of the Amiel-Tison neurologic assessment for the term neonate or at 40 weeks corrected age. Pediatr Neurol 2002;27(3):196-212.

42. Campbell S. 4D, or not 4D: that is the question. Ultrasound Obstet Gynecol 2002;19(1):1-4.

43. Campbell S. Prenatal ultrasound examination of the secondary palate. Ultrasound Obstet Gynecol 2007;29(2):124-127.

44. Luedders DW, Bohlmann MK, Germer U, Axt-Fliedner R, Gembruch U, Weichert J. Fetal micrognathia: objective assessment and associated anomalies on prenatal sonogram. Prenat Diagn 2011;31(2):146-151.

45. Terzis JK, Anesti K. Developmental facial paralysis: a review. J Plastic Reconst Aesthetic Surg 2011;64:e1318-e1333.

46. Lee W, McNie B, Chaiworapongsa T, Conoscenti G, Kalache KD, Vettraino IM, Romero R, Comstock CH. Threedimensional ultrasonographic presentation of micrognathia. J Ultrasound Med 2002;21(7):775-781. 
47. Borenstein M, Persico N, Kagan KO, Gazzoni A, NIcolaides $\mathrm{KH}$. Frontomaxillary facial angle in screening for trisomy 21 at $11+0$ to $13+6$ weeks. Ultrasound Obstet Gynecol 2008;32(1): 5-11.

48. Suttie M, Foroud T, Wetherill L, Jacobson JL, Molteno CD, Meintjes EM, Hoyme HE, Khaole N, Robinson LK, Riley EP, Jacobson SW, Hammond P. Facial dysmorphism across the fetal alcohol spectrum. Pediatrics 2013;131:e779-e788.

49. Neugebauer R, Kline J, Stein Z, Shrout P, Warburton D, Susser M. Association of stressful life events with chromosomally normal spontaneous abortion. Am J Epidemiol 1996;143(6): 588-596.

50. Fenster L, Schaefer C, Mathur A, Hiatt R, Pieper C, Hubbard A, Swan SH. Psychologic stress in the workplace and spontaneous abortion. Am J Epidemiol 1995;142(11):1176-1183.

51. Green M, Rani C, Joshi A, Soto-Pina A, Martinez P, Frazer A, Morilak D. Prenatal stress induces long-term stress vulnerability, compromising stress response systems in the brain and impairing extinction of conditioned fear after adult stress. Neuroscience 2011 Sept;192:438-451.

52. Schreier N, Moltchanova E, Blomstedt P, Kajantie E, Eriksson J. Prenatal exposure to wartime stress-long-term effect on coronary heart disease in later life. Ann Med 2011;43(7): 555-561.
53. Reissland N, Aydin E, Francis B, Exley K. Laterality of fetal self-touch in relation to maternal stress. Laterality doi: 10.1080/1357650X.2014.920339.

54. Talic A, Kurjak A, Ahmed B, Stanojevic M, Predojevic M, Kadic AS, Di Renzo GC. The potential of 4D sonography in the assessment of fetal behavior in high-risk pregnancies. J Matern Fetal Neonat Med 2011;24(7):948-954.

55. De Jong-PleijEAP, RibbertLSM,Pistorius LR, Tromp E, Mulder EJH, Bilardo CM. Three-dimensional ultrasound and maternal bonding, a third trimester study and a review. Prenat Diagn 2013;33(1):81-88.

56. Herrero JR, Comas C, Azumendi G, Alonso, I, Romero M, Anderica J, Calvo A, Narbona I, Rius F. Maternal attitudes towards three- and four-dimensional ultrasound during pregnancy. Ultrasound Obstet Gynecol 2006;28(4):579-580.

57. Krubitzer L, Kahn DM. Nature versus nurture revisited: an old idea with a new twist. Progress in Neurobiology 2003;70:33-52.

58. Nicoletto SF, Rinaldi A. In the womb's shadow. EMBO Reports 2011;12(1):30-34.

59. Myowa-Yamakoshi M, Takeshita H. Do human fetuses anticipate self-oriented actions? A study by four-dimensional (4D) ultrasonography. Infanc, 2006;10(3):289-301.

60. Di Pietro JA, Caulfield LE, Costigan KA, Merialdi M, Nguyen RHN, Zavaleta N, Gurewitsch ED. Fetal neurobehavioral development: A tale of two cities. Dev Psychol 2004;40(3):445-456. 